DOI 10.37882/2223-2982.2021.05-2.08

\title{
ТЕОРЕТИЧЕСКИЕ ОСНОВЫ МОДЕЛИРОВАНИЯ СПОРТИВНОЙ ТРЕНИРОВКИ СТАЙЕРОВ С ИСПОЛЬЗОВАНИЕМ ЦИФРОВЫХ ТЕХНОЛОГИЙ
}

\section{THEORETICAL BASES FOR MODELING SPORTS TRAINING OF STAYERS USING DIGITAL TECHNOLOGIES}

D. Herzen

Summary: This article focuses on solving the following goal: strategies for integrating digital technologies in sports training for long-term athletes. The study was conducted based on the method of analysis of scientific literature, digital devices and applications (Training Peaks, Strava, Endomondo, Runkeeper, Google Fit, Nike + Run Club, etc.). The author defines sports smart training. The article establishes and describes three strategies for the implementation of sports smart training for marathon runners. The first strategy assumes that the athlete interacts with the coach in person, therefore, it is advisable to use digital technologies at the stages of implementation, monitoring and evaluation, indirectly at the planning stage, since, considering sports training on a cyclic scale, it is obvious that sports training is adapted at the stage of subsequent planning, taking into account the assessment carried out. The second strategy is focused on replacing the coach with a smart coach. In this case, the smart coach is delegated the authority to plan, control and evaluate training activities. The third strategy involves independent training by an athlete who uses digital technologies primarily at the stages of implementation, control (wearable sensors, sports watches), indirectly at the stages of planning and assessment (smart applications), but the athlete makes decisions independently.

Keywords: sports, marathon running, digital running training technology, sports training, sports smart training.

\author{
Герцен Дмитрий Александрович \\ аспирант, ГАОУ ВО г. Москвы «Московский городской \\ педагогический университет» \\ gertsen.dmitry@gmail.com
}

Аннотация: Данная статья посвящена решению следующей цели: стратегий внедрения цифровых технологий в спортивную тренировку стайеров. Исследование проводилось на основе метода анализа научной литературы, цифровых устройств и приложений (Training Peaks, Strava, Endomondo, Runkeeper, Google Fit, Nike+ Run (lub и др.). Автор дает определение спортивной смарттренировки. В статье установлены и охарактеризованы три стратегии реализации спортивной смарт-тренировки марафонцев. Первая стратегия предполагает, что спортсмен взаимодействует с тренером очно, следовательно, целесообразно использовать цифровые технологии на этапах реализации, контроля и оценки, опосредованно на этапе планирования, поскольку, рассматривая спортивную тренировку в циклическом масштабе, очевидным является адаптация спортивной тренировки на этапе последующего планирования с учетом проведенной оценки. Вторая стратегия ориентирована на замену тренера смарт-коучем. В этом случае смарт-коучу делегируются полномочия планирования, контроля и оценки тренировочной деятельности. Третья стратегия предполагает самостоятельные тренировки спортсменом, который использует цифровые технологии прежде всего на этапах реализации, контроля (носимые датчики, спортивные часы), опосредованно на этапах планирования и оценки (смарт-приложения), однако решения спортсмен принимает самостоятельно.

Ключевые слова: спорт, марафонский бег, цифровые технологии тренировки бега, спортивная тренировка, спортивная смарт-тренировка.

\section{Введение}

Б ыстрое развитие информационных технологий оказало влияние практически на все сферы нашей жизни. Компьютеры, смартфоны, умные часы и другие мобильные и широко распространенные технологии меняют способы нашей деятельности и наше восприятие внешнего мира. Нет сомнений в том, что наша цивилизация должна адаптироваться ко многим изменениям, которые обусловлены развитием современных технологий.

Спортивные тренировки не являются исключением, напротив, представляют собой перспективную область исследований, ориентированную, с одной стороны, на совершенствование спортивных результатов и достижений, с другой стороны, на развитие современных технологий. Данный процесс следует понимать как взаимообусловленный. Кроме того, актуальность развития области диджитализации спортивной тренировки значительно повышается в контексте тенденции роста участия спортсменов-любителей в массовых спортивных мероприятиях, среди которых лидируют соревнования по бегу. В этом случае цифровые технологии тренировки бега представляют собой качественную альтернативу поддержки тренера, поскольку далеко не все спортсмены-любители могут нанять тренера из-за множества препятствий, например, финансовых. 


\section{Материалы и методы}

В данном контексте уместным является обращение к концепту спортивной смарт-тренировке (Smart Sport Training (SST)). Основываясь на исследованиях I. Fister, I.Jr. Fister, D. Fister [7], M. Héder [8], A. Rajšp, I.Jr. Fister [10], спортивную смарт-тренировку в настоящем исследовании возможно определить как тип спортивной тренировки, в которой используются носимые устройства, датчики и устройства Интернета вещей (IoT), а также интеллектуальные методы и инструменты анализа данных для повышения эффективности тренировок и / или снижения рабочей нагрузки при сохранении или повышении тренировочной производительности. Это означает, что реализации спортивной смарт-тренировки варьируются от простых задач, таких как внедрение в тренировочную деятельность носимых устройств, осуществление интеллектуального анализа данных сеанса, до гораздо более сложных: реализация функции смарт-коуча, где тренер заменяется умным агентом, который управляет всеми аспектами обучения, кроме собственно выполнения предложенных упражнений для обучаемого. Снижение нагрузки может касаться как спортсмена, так и его тренера. Для спортсмена улучшенный план тренировок означает, что он может достичь лучших результатов при том же или даже меньшем количестве тренировок, а для его тренера это означает, что с помощью цифровых технологий для тренировки бега можно автоматизировать части его тренировочной программы.

Область исследований, посвященных интеллектуальным методам анализа данных в области спортивной тренировки, в настоящее время становится очень популярной. Вместе с тем, несмотря на интерес исследователей к проблематике, круг источников крайне ограничен.

Спортивная тренировка - это непрерывный процесс взаимодействия между спортсменом и его тренером. Это педагогический процесс, в котором тренер выполняет роль учителя, руководителя деятельности спортсмена и организатора его тренировок. Тренировочные упражнения - это четко определенные задачи, которые требуют физических усилий и нацелены на улучшение спортивных результатов обучаемого. Несколько тренировочных упражнений объединяются в полные блоки, называемые тренировочными занятиями. Конечная цель спортивной тренировки - совершенствование способностей спортсмена, достижение их природного потенциала. Опираясь на концепцию спортивной тренировки Л.П. Матвеева, непрерывный процесс обучения можно кратко разбить на следующие четыре этапа [1].

Этап планирования предполагает определение правильных единиц упражнений. Цикл спортивных тренировок ориентирован на календарь соревнований. На этом этапе тренер составляет график упражнений для спортсмена.
Этап реализации нацелен на выполнение спортсменом запланированных упражнений в комплексе спортивной тренировки. Роли тренера на этом этапе сводится к проведению психофизической оценки спортсмена перед тренировкой, мониторингу интенсивности тренировки. На этом этапе фиксируются данные показателей, необходимые для дальнейшего анализа.

Этап контроля предполагает сравнение упражнений, фактически выполняемых спортсменом, с запланированными упражнениями. В беговых дисциплинах можно проводить биометрический анализ результатов.

Этап оценки содержит измерение производительности спортсмена. Существует два вида оценок: оценка отдельной тренировочной нагрузки (краткосрочный анализ эффективности) и оценка общей нагрузки цикла тренировки (долгосрочный анализ эффективности). Оценка - это сравнение между поставленными целями и достигнутыми результатами, а также количеством запланированных и фактически выполненных упражнений.

Поскольку спортивная тренировка - это деятельность, по крайней мере, двух сторон, а именно тренера и спортсмена, можно использовать различные цифровые технологии, чтобы помочь тренеру в принятии решений, или полностью заменить его, использовав смарт-коуча. Это позволяет спортсмену выбирать из множества возможных режимов тренировок без необходимости нанимать человека для помощи в тренировках.

\section{Результаты}

Использование цифровых технологий тренировки бега возможно в рамках трех стратегий.

Первая стратегия предполагает, что спортсмен взаимодействует с тренером очно, следовательно, целесообразно использовать цифровые технологии на этапах реализации, контроля и оценки, опосредованно на этапе планирования, поскольку, рассматривая спортивную тренировку в циклическом масштабе, очевидным является адаптация спортивной тренировки на этапе последующего планирования с учетом проведенной оценки.

В качестве цифровых технологий, успешно выполняющих поддерживающую функцию спортивной тренировки при непосредственном взаимодействии спортсмена с тренером, рассматриваются прежде всего носимые датчики, спортивные часы для бега, а также приложения и онлайн-сервисы, позволяющие фиксировать планирование и производить автоматизированную оценку результатов в сравнении с результатами других тренировок (например, в качестве наиболее зарекомендовавших себя приложений следует отметить Strava, Endomondo, Runkeeper, Google Fit, Nike+ Run Club; в ка- 
честве онлайн-сервисов - Strava, Google Fit, Nike+ Run Club и др.).

Большими возможностями на этапе оценки обладает технология цифрового двойника, которая успешно используется в приложении Strava. В науке содержатся примеры продуктивной оценочной деятельности в структуре спортивной тренировки марафонцев посредством цифровых технологий. В исследовании I. Fister, D. Fister, S. Deb, U. Mlakar, J. Brest описывается метод определения оптимальной скорости на марафонской дистанции посредством изучения временных потерь на основе данных GPS [6]. В работе научного коллектива I. Fister, D. Fister, S. Deb, U. Mlakar, J. Brest представлен метод анализа результатов марафонцев, основанные на изучении частоты сердечных сокращений. Авторы приводят 25 различных стратегий, определенных алгоритмом дифференциальной эволюции, по сокращению дефицита времени на каждом километре дистанции [5].

Вторая стратегия ориентирована на замену тренера смарт-коучем. В этом случае смарт-коучу делегируются полномочия планирования, контроля и оценки тренировочной деятельности. Приложения Strava и Training Peaks предлагают такой вариант тренировочной деятельности для пользователей. F. Buttussi, L. Chittaro еще в 2008 году продемонстрировали эффективность системы спортивных тренировок, курируемых смарт-коучем. Авторы являются разработчиками персональной тренировочной системы МОРЕТ, базирующейся на использовании носимых датчиков и применении контекстно-зависимых и адаптивных рекомендаций, формируемых на основе технологии цифрового двойника, а также информации, полученной от спортивного физиолога [3].

Ключевая проблема, которая возникает при использовании данной стратегии, заключается в том, что спортсмен может не реализовать предписанную смарт-коучем систему тренировок. Исследование С.А. Clermont, L. Duffett-Leger, B.A. Hettinga, R. Ferbe подтверждает данное утверждение. Авторы проведи опрос бегунов-марафонцев, как профессионалов, так и любителей; им удалось установить, что около половины респондентов изменяли самостоятельно тренировочный процесс, а около трети строили тренировку, не обращая внимания на рекомендации смарт-коуча [4]. Очевидно, что проблема использования цифровых технологий при данной стратегии заключается в мотивации и навыков целеполагания спортсменов. Проблемной зоной в этом плане выступает этап реализации, который часто не коррелирует с этапом планирования и, соответственно, контроля.

Разработчики Training Peaks заявляют, что данная система достаточно адаптивна и способна подстраиваться под изменяющиеся потребности пользователя, однако вполне очевидно, что постоянное отступление от тренировочного плана не является эффективной стратегией тренировки.

Третья стратегия предполагает самостоятельные тренировки спортсменом, который использует цифровые технологии прежде всего на этапах реализации, контроля (носимые датчики, спортивные часы), опосредованно на этапах планирования и оценки (смарт-приложения), однако решения спортсмен принимает самостоятельно.

A. Rajšp, I.Jr. Fister пишут, что потенциал спортивной смарт-тренировки достаточно высок на всех ее этапах, однако авторы утверждают, что наиболее разработанной практикой является применение цифровых технологий спортсменом на этапе реализации спортивной тренировки, поскольку современные носимые устройства позволяют вести запись фактических данных во время тренировочного процесса. Некоторые многоцелевые носимые устройства могут использоваться в различных областях, обусловливающих результативность спортивной тренировки, поскольку содержат датчики для отслеживания сна, мониторинга сердечного ритма, питания, GPS и т. д. [10]. Исследования, относящиеся к этапу контроля спортивной тренировки, тесно связаны с исследованием этапа реализации, как правило, представляют собой изучение средств для записи данных тренировки.

Например, в исследовании P. Lopez-Matenci, J.V. Alonso, F.J. Gonzalez-Castano, J.L. Sieiro, J.J. Alcaraz описываются результаты внедрения системы искусственного интеллекта применительно к занятиям беговыми видами спорта с поддержкой персонализированной обратной связи для спортсменов в режиме реального времени. Данная система обеспечивает обратную связь в реальном времени (с точностью до 70\%) в зависимости от местности, температуры и уклона трассы [9]. С. Strohrmann, H. Harms, G. Troster резюмируют результаты испытания минимального набора из двух датчиков ускорения, прикрепленных к ступне и бедру спортсмена, для определения кинематических характеристик опытных и неопытных бегунов [11].

Исследования использования цифровых технологий на этапе оценки были в основном связаны со сравнением тренировочных данных и результатами соревнований (например, [6, 5]).

Наименьшее количество исследований проводится применительно к этапу планирования тренировок, где все еще преобладают настоящие тренеры-люди. Например, K. Brzostowski, J. Drapała, A. Grzech, P. Świątek описывают результаты исследования планирования опти- 
мальной скорости бега спортсмена посредством оценки физических усилий, необходимых для каждой части соревнований и тренировок. Данная работа является примером разработки адаптивной системы поддержки принятия решений для спортивной тренировки на основе оценки оптимальной скорости бега спортсмена [2].

Реализация третьей стратегии спортивной тренировки бегунов-марафонцев, помимо зоны риска, связанной с мотивацией, выявленной при анализе второй стратегии, связана еще с одной зоной риска, находящейся на этапах оценки и планирования. Поскольку в настоящее время отсутствуют в широком доступе качественные цифровые инструменты, позволяющие реализовать данные этапы спортивной тренировки бегунов, их применение целиком и полностью зависит от спортивной компетентности стайера.

\section{Обсужмение}

В последние годы исследования спортивной смарттренировки заметно активизировались, однако, опираясь на авторитетное мнение A. Rajšp, I.Jr. Fister, следует отметить, что ни один из реализуемых подходов ни одного из обучающих приложений бегу не проходит необходимый порог уровня готовности технологии TRL. Это может показаться удивительным, поскольку многие приложения широко используются на устройствах Android, например, MyFitnessPal, Endomondo Running and Walking, Google Fit и устройства экосистемы Apple, такие как Apple Health. Наивысшим достигнутым уров- нем TRL были зафиксированы на этапах реализации и контроля [10].

\section{Зак^ючение}

Таким образом, исследование теоретических основ моделирования индивидуального тренировочного процесса марафонцев с использованием современных цифровых технологий позволило выделить три стратегии. Субъектами первой стратегии являются тренер и спортсмен, второй стратегии - смарт-коуч и спортсмен, третьей стратегии - только спортсмен. Наиболее проработанными лакунами применения цифровых технологий при реализации всех трех стратегий являются этапы реализации и контроля спортивной тренировки в основном благодаря носимым датчикам, спортивным часам и приложениям для бега. Наиболее уязвимыми для стратегий, исключающих реального тренера, являются этапы планирования и оценки. Зонами риска для второй и третьей стратегий выступает связь этапов планирования и реализации, а также реализации и контроля, чему причиной является недостаточный уровень мотивации и целеполагания спортсмена. В третьей стратегии обнаруживается еще одна зона риска - этапы оценки и планирования, которые детерминированы недостаточным уровнем спортивной компетентности бегуна-любителя. В качестве возможного инструмента преодоления данных рисков можно рассматривать самообразование и публичное целеполагание в формате участия спортсменов в специализированных социальных сетях и сообществах по бегу.

\section{ЛИТЕРАТУРА}

1. Матвеев, Л.П. Основы спортивной тренировки / Л.П. Матвеев. - Тбилиси: Ганатлеба, 1987. - 366 с.

2. Brzostowski, K., Drapała, J., Grzech, A., Świątek, P. Adaptive decision support system for automatic physical effort plan generation-data-driven approach / K. Brzostowski, J. Drapała, A. Grzech, P. Świątek // Cybernetics and Systems. - 2013. - Vol. 44 (2-3). - P. 204-221.

3. Buttussi, F., Chittaro, L. MOPET: A context-aware and user-adaptive wearable system for fitness training / F. Buttussi, L. Chittaro // Artificial Intelligence in Medicine. - 2008. - Vol. 42. - P. 153-163.

4. Clermont, C.A., Duffett-Leger, L., Hettinga, B.A., Ferber, R. Runners' Perspectives on 'Smart' wearable technology and its use for preventing injury / C.A. Clermont, L. Duffett-Leger, B.A. Hettinga, R. Ferber // International Journal of Human-Computer Interaction. - 2019. \#1. - P. 1-10.

5. Fister, I., Fister, D., Deb, S., Mlakar, U., Brest, J. Making up for the deficit in a marathon run / I. Fister, D. Fister, S. Deb, U. Mlakar, J. Brest // Proceedings of the 2017 International Conference on Intelligent Systems, Metaheuristics \& Swarm Intelligence - ISMSI'17, Hong Kong, China, 25-27 March 2017. - ACM Press: New York, NY, USA, 2017. - P. 11-15.

6. Fister, I., Fister, D., Deb, S., Mlakar, U., Brest, J. Post hoc analysis of sport performance with differential evolution. / I. Fister, D. Fister, S. Deb, U. Mlakar, J. Brest / Neural Computing and Applications. - Springer: Cham, Switzerland, 2018. - P. 1-10.

7. Fister, I., Fister, I. Jr., Fister, D. Computational Intelligence in Sports / I. Fister, I.Jr. Fister, D. Fister. - Springer: Cham, Switzerland, 2019. - 298 p.

8. Héder, M. From NASA to EU: The Evolution of the TRL Scale in Public Sector Innovation / M. Héder // The Innovation Journal: The Public Sector Innovation Journal. 2017. - Vol. 22(2), article 3. - P. 1-23.

9. Lopez-Matenci, P., Alonso, J.V., Gonzalez-Castano, F.J., Sieiro, J.L., Alcaraz, J.J. Ambient intelligence assistant for running sports based on k-NN classifiers / P. LopezMatenci, J.V. Alonso, F.J. Gonzalez-Castano, J.L. Sieiro, J.J. Alcaraz // Proceedings of the 3rd International Conference on Human System Interaction. - Rzeszow, Poland, 2010. - P. 605-611.

10. Rajšp, A., Fister, I.Jr. A Systematic Literature Review of Intelligent Data Analysis Methods for Smart Sport Training / A. Rajšp, I.Jr. Fister // Applied Sciences. - 2020. - Vol. 10(9): 3013. - doi.org/10.3390/app10093013 
11. Strohrmann, C., Harms, H., Troster, G. What Do Sensors Know about Your Running Performance? / C. Strohrmann, H. Harms, G. Troster / Proceedings of the 2011 15th Annual International Symposium on Wearable Computers, San Francisco, CA, USA, 12-15 June 2011. - San Francisco, CA, USA, 2011. - P. $101-104$.

(с) Герцен Дмитрий Александрович (gertsen.dmitry@gmail.com).

Журнал «Современная наука: актуальные проблемы теории и практики»

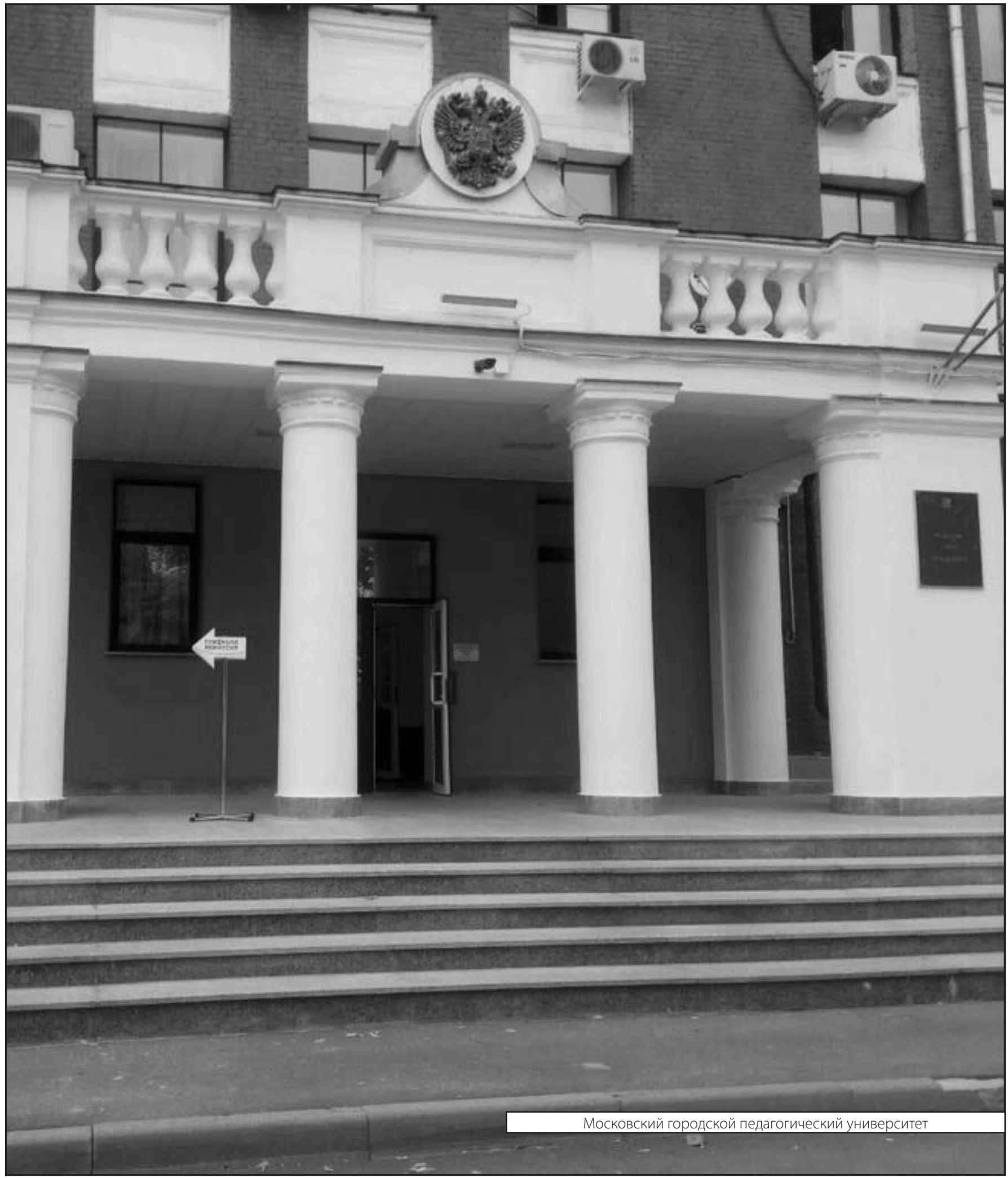

